\title{
Beyond bones: The relevance of variants of connective tissue (hypermobility) to fibromyalgia, ME/CFS and controversies surrounding diagnostic classification: an observational study
}

\author{
Authors: Jessica A Eccles, ${ }^{\mathrm{A}}$ Beth Thompson, ${ }^{\mathrm{B}}$ Kristy Themelis, ${ }^{\mathrm{C}}$ Marisa L Amato, ${ }^{\mathrm{D}}$ Robyn Stocks, ${ }^{\mathrm{B}}$ Amy Pound, ${ }^{\mathrm{E}}$ \\ Anna-Marie Jones, ${ }^{F}$ Zdenka Cipinova, ${ }^{G}$ Lorraine Shah-Goodwin, ${ }^{G}$ Jean Timeyin, ${ }^{G}$ Charlotte R Thompson, ${ }^{H}$ \\ Thomas Batty, ${ }^{\mathrm{I}}$ Neil A Harrison, ${ }^{3}$ Hugo D Critchley ${ }^{\mathrm{K}}$ and Kevin A Davies ${ }^{\mathrm{L}}$
}

\section{Background}

Fibromyalgia and myalgic encephalomyelitis / chronic fatigue syndrome (ME/CFS) are poorly understood conditions with overlapping symptoms, fuelling debate as to whether they are manifestations of the same spectrum or separate entities. Both are associated with hypermobility, but this remains significantly undiagnosed, despite impact on quality of life.

Objective

We planned to understand the relevance of hypermobility to symptoms in fibromyalgia and ME/CFS.

Method

Sixty-three patient participants presented with a confirmed diagnosis of fibromyalgia and/or ME/CFS; 24 participants were healthy controls. Patients were assessed for symptomatic hypermobility.

Authors: ${ }^{\text {A }}$ linical senior lecturer, Brighton and Sussex Medical School, Falmer, UK, Brighton and Sussex University Hospitals NHS Trust, Brighton, UK and Sussex Partnership Foundation NHS Trust, Brighton, UK; ${ }^{B}$ medical student, Brighton and Sussex Medical School, Falmer, UK; ${ }^{C}$ post-doctoral research fellow, Brighton and Sussex

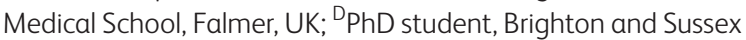
Medical School, Falmer, UK; Eresearch assistant, Brighton and Sussex Medical School, Falmer, UK and Sussex Partnership Foundation NHS Trust, Brighton, UK; F statistician, Sussex Partnership Foundation NHS Trust, Brighton, UK; ${ }^{G}$ research nurse, Brighton and Sussex University Hospitals NHS Trust, Brighton, UK; ${ }^{\mathrm{H}}$ senior lecturer in rheumatology, Brighton and Sussex University Hospitals NHS Trust, Brighton, UK and Brighton and Sussex Medical School, Falmer, UK; ' Imedical education fellow, Brighton and Sussex Medical School, Falmer, UK and Brighton and Sussex University Hospitals NHS Trust, Brighton, UK; ${ }^{\text {}}$ professor, Brighton and Sussex Medical School, Falmer, UK and Cardiff University Brain Research Imaging Centre, Cardiff, UK; ${ }^{\mathrm{K}}$ chair in psychiatry, Brighton and Sussex Medical School, Falmer, UK, University of Sussex, Falmer and Sussex Partnership Foundation NHS Trust, Brighton, UK; ' 'chair in medicine, Brighton and Sussex University Hospitals NHS Trust, Brighton, UK and Brighton and Sussex Medical School, Falmer, UK

\section{Results}

Evaluations showed exceptional overlap in patients between fibromyalgia and ME/CFS, plus $81 \%$ met Brighton criteria for hypermobility syndrome (odds ratio 7.08 ) and $18 \%$ met 2017 hypermobile Ehlers-Danlos syndrome (hEDS) criteria. Hypermobility scores significantly predicted symptom levels.

Conclusion

Symptomatic hypermobility is particularly relevant to fibromyalgia and ME/CFS, and our findings highlight high rates of mis-/underdiagnosis. These poorly understood conditions have a considerable impact on quality of life and our observations have implications for diagnosis and treatment targets.

KEYWORDS: hypermobility, pain, fatigue, ME/CFS, fibromyalgia

DOI: $10.7861 /$ clinmed.2020-0743

\section{Introduction}

Fibromyalgia and myalgic encephalomyelitis / chronic fatigue syndrome (ME/CFS) have overlapping symptoms. All physicians will be facing the challenge of assessing possible 'post-viral fatigue' in the wake of COVID-19. Fibromyalgia is a common, poorly understood, complex musculo-skeletal disorder that affects around $5 \%$ of the UK population. ${ }^{1}$ It is polysymptomatic yet marked primarily by chronic widespread pain, typically believed to be non-inflammatory in nature. In addition, patients frequently report extra-musculoskeletal symptoms, including both physical and emotional fatigue with subjective cognitive dysfunction ('fibrofog'). ${ }^{2}$ For quality of life, patients with fibromyalgia score significantly lower than the general population, or patients with other musculo-skeletal disorders (such as rheumatoid arthritis, osteoarthritis and systemic lupus erythematosus) on all eight health status domains. ${ }^{2,3}$ Pain, fatigue, subjective cognitive impairment and psychological disturbance (eg depression and anxiety) have greatest impact on quality of life. Thus, pain and fatigue are identified as among the most disabling symptoms associated 
with fibromyalgia. Relatedly, pain is also frequently reported in ME/CFS. ${ }^{4}$ Complexity of understanding their origins may have contributed at times to consideration of both fibromyalgia and ME/ CFS as functional somatic, medically unexplained or somatisation disorders. The presence of shared symptoms in brain and body has also fuelled debate as to whether fibromyalgia and ME/CFS are manifestations of the same spectrum disorder or separate clinical entities. ${ }^{5,6}$ Although the dominant symptom of fibromyalgia is pain and ME/CFS is disabling fatigue or post-exertional malaise, patients with either diagnosis typically experience pain (including hyperalgesia and central sensitisation), fatigue, sleep problems and cognitive difficulties. ${ }^{6}$ Altered inflammatory markers and dysautonomia are reported in both fibromyalgia and ME/CFS, calling into question their perceived status as 'functional' disorders. ${ }^{7-10}$ Moreover, it is increasingly recognised that fibromyalgia and ME/ CFS are associated with joint hypermobility, an expression of variant connective tissue. ${ }^{11-14}$

Joint hypermobility (a feature of Ehlers-Danlos syndromes (EDS)) is common and associated with many extra-articular features. ${ }^{15,16}$ Internationally, there is little agreement on the precise definition of measurement and hypermobility, nor the point at which it constitutes a disorder. In 2017, a consensus group sought to re-classify Ehlers-Danlos syndrome - hypermobility type (EDS$\mathrm{HT}$ ). As a consequence, the terms hEDS (hypermobile EDS) and hypermobility spectrum disorder (HSD) now refer to symptomatic joint hypermobility. ${ }^{17}$ An earlier term, joint hypermobility syndrome (diagnosed by revised Brighton criteria) was made redundant and was previously deemed indistinguishable from EDS-HT. ${ }^{18,19}$ However, one important distinction is that the revised Brighton criteria took into account historical joint hypermobility, whereas the newer criteria for hEDS rely on age and gender specific hypermobility cut-offs. There are commonalities in these criteria (including importance of widespread pain), but important differences in significance of Beighton (joint hypermobility) scoring, precise consideration of connective tissue and associated features and evaluation of family history (supplementary material S1). A broader concept of a HSD was introduced to describe the continuum that encompasses patients with symptomatic joint hypermobility through to people who do not meet full criteria for hEDS or related syndromes associated with joint hypermobility. However, this notion of a spectrum also remains controversial. Since there are no genetic tests available for hEDS, a clinical diagnostic checklist is available and consistent with the 2017 consensus criteria. ${ }^{17}$ Further research is required to validate clinical diagnostic criteria and international diagnostic codes, which presently are not consistent with newer nomenclature. In adults with hEDS/HSD, symptoms (including pain, fatigue, dysautonomic symptoms, poor coordination, attention/concentration deficits and low quality of life in general) can be also classified according to severity, reflecting neither the old nor new criteria for hEDS. ${ }^{20}$ Debate regarding diagnostic classification has intensified following a report on the prevalence of EDS and joint hypermobility syndrome (JHS) in primary care that suggests these diagnoses cannot be considered rare. ${ }^{21}$ Moreover, symptomatic hypermobility has considerable impact on quality of life, at least comparable or greater than those with other rheumatological diseases. ${ }^{22}$

The morbidity of hEDS/HSD to patients is most commonly expressed as complaints to clinicians of chronic musculoskeletal pain, headache and fatigue. These symptoms impair physical activity and quality of life, common to both fibromyalgia and
ME/CFS. In rheumatology clinics, symptomatic hypermobility is estimated to contribute to nearly half of all outpatient appointments, but is only recognised one out of 19 times. $^{14}$ Under-diagnosis of hEDS/HDS may exacerbate potentially disabling symptoms, complications and comorbidities, including autonomic dysfunction, fibromyalgia and ME/CFS. ${ }^{10,23}$ Enhanced recognition of symptomatic hypermobility is essential to improve function and quality of life in a patient group, whose symptoms are typically poorly understood and frequently overlooked..$^{14}$ Accurate phenotyping of hEDS/HDS is needed for research, but it is also critical to effective multidisciplinary clinical management, from provision of accurate prognostic advice and identification of comorbidity to the offer of multiprofessional support and delivery of effective therapies. The relevance extends beyond rheumatology to other clinical contexts (eg cardiology, paediatrics, neurology, gastroenterology, pain management and mental health settings).

\section{Aims of the study}

The objective of this study was to understand the relevance of symptomatic hypermobility and related connective tissue variants to the expression of symptoms in fibromyalgia and ME/CFS. The study systematically tests associations between the diagnostic criteria for pain and fatigue symptom expression and further examines if specific subfactors within the diagnostic classifications of hypermobility predict clinical presentations.

\section{Methods}

This study is part of larger study investigating mechanisms of chronic pain and fatigue in fibromyalgia and ME/CFS (https://doi.org/10.1186/ ISRCTN78820481) in adults. Here, we focus on the relevance of symptomatic hypermobility. All participants (patient participants and healthy controls) provided written informed consent and were compensated for their participation.

\section{Patient and public involvement}

The study idea was formed after patients and prior research participants discussed their lived experience of fibromyalgia and ME/CFS with the clinicians involved in the initial grant application. Lay members of Arthritis Research UK critically reviewed the proposal and their suggestions incorporated into the proposal. A formal patient and public involvement panel reviewed the study after funding was obtained and advised on the study protocol and design, strategies for recruitment reflecting their priorities, experiences and preferences. Patients and public assessed the burden of testing and the time required to participate in the study.

Patients and public were involved in the promotion of the study through local support groups for patients living with fibromyalgia and ME/CFS.

Ethical approval was obtained from London - Brighton \& Sussex Research Ethics Committee (REF:17/LO/0845). Patient participants with a clinical diagnosis of either fibromyalgia and/or ME/CFS were invited to be screened for the study.

To be included in the study, patients were required to either meet the American College of Rheumatology (ACR; 2010) diagnostic criteria for fibromyalgia and/or both the Canadian and Fukada 


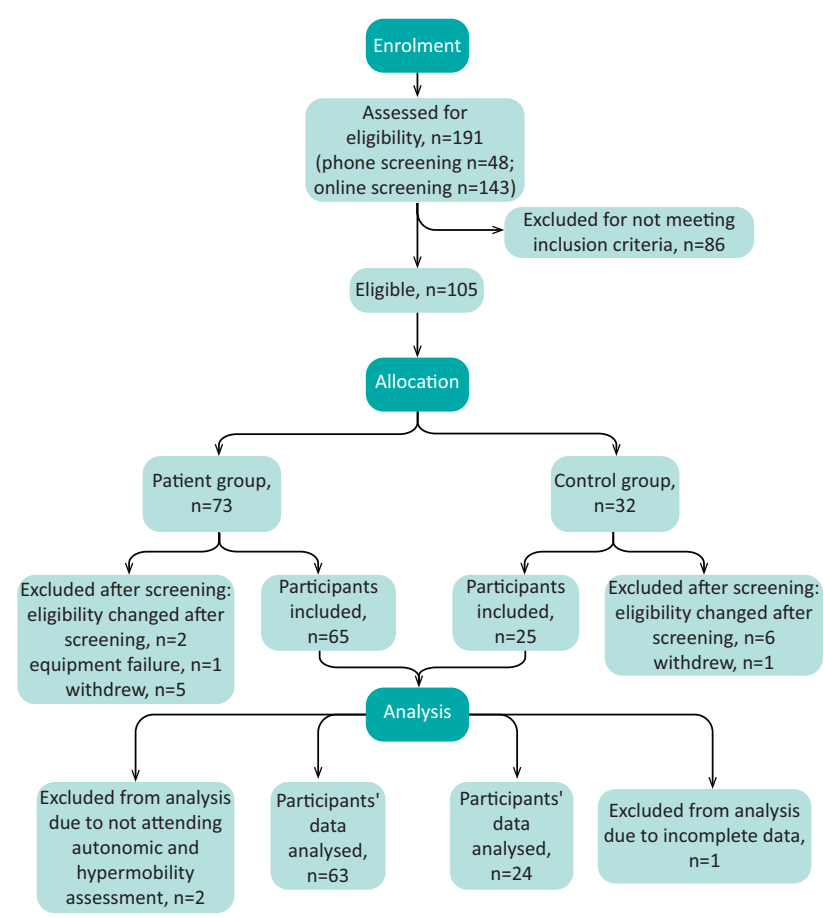

Fig 1. CONSORT diagram showing participant recruitment and flow through the study.

criteria for ME/CFS as assessed at screening. Patients were recruited by advertisement from local support organisations for fibromyalgia and ME/CFS, local rheumatology clinics and via social media and bulletin boards. Inclusion criteria for healthy controls were a score no more than $3 / 10$ on a pain visual analogue scale; $a$ score of less than 36 on the fatigue severity scale and not meeting either the criteria for ACR diagnostic criteria for fibromyalgia nor the Canadian and Fukada criteria for ME/CFS; and be free of major psychiatric or neurological illness.

Healthy controls were also recruited via social media and bulletin boards. Neither patients nor controls were recruited/selected or screened on the basis of hypermobility and hypermobility assessors where unaware of clinical or screening disease specific research diagnostic information.

All participants who responded to advertisements were provided with more information. If they were willing to continue, there were screened via telephone or via anonymised online questionnaires for eligibility. Fig 1 shows a CONSORT diagram detailing participant flow. Consenting participants completed a series of baseline questionnaires measuring subjective pain (McGill pain short form questionnaire); fatigue (fatigue impact, modified fatigue impact scale); brain-fog (mental clutter scale) and interoceptive sensibility (Porges body perception questionnaire). ${ }^{24}$ Demographic data was also collected and participants were assessed for presence of symptomatic hypermobility and variants of connective tissue by trained clinicians using the revised Brighton criteria and the 2017 hEDS classification criteria. ${ }^{14,17}$ It was not possible to determine the last item in criterion $2 \mathrm{~A}$ (aortic root dilatation with Z-score $>+2$ ) as participants did not undergo echocardiography as part of the study protocol. Given the association between variant connective tissue and easy
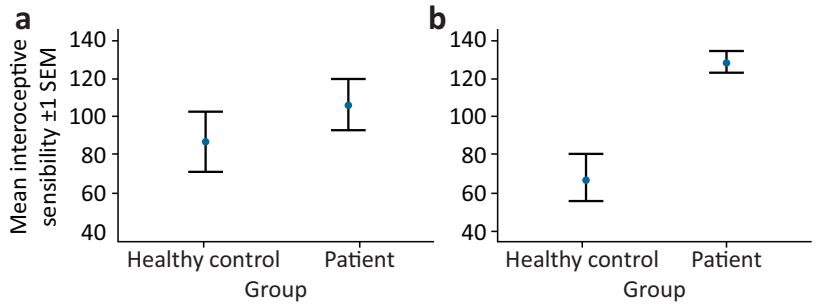

Fig 2. The significant interaction of meeting Brighton criteria for joint hypermobility syndrome on the association between patient group and interoceptive sensibility. a) Does not meet Brighton criteria for joint hypermobility syndrome. b) Meets Brighton criteria for joint hypermobility syndrome. SEM = standard error of the mean.

bruising, all participants where asked about this phenomenon independently. ${ }^{25}$ In total, 24 healthy controls were assessed alongside 63 patient participants. Where possible, controls were matched to age and gender of patient participants.

\section{Data analysis and statistical methods}

Each symptomatic hypermobility diagnostic classification (revised Brighton criteria for JHS; hEDS 2017 consensus criteria) were entered as binary variables. Further binary variables explored categorical elements of the different criteria. The Beighton scale and total number of major and minor criteria endorsed were expressed as scale data. Differences between groups in age and assessment outcomes were assessed via independent sample t-tests. Differences in sex and associations between groups were assessed via chi squared test. Binary logistic regression was used to derive prediction models for categorical variables, their odds ratios and their confidence intervals where appropriate. Univariate analyses (general linear model) were used to determine interactions. Spearman correlations were used to explore correlations with Beighton score. Hypothesis tests were considered statistically significant for $p$ values $<0.05$ and all analyses were carried out using SPSS version 25 for Mac.

\section{Results}

\section{Patient characteristics and diagnostic groups}

Twenty-four healthy controls (nine male) and patients (12 male) did not differ significantly in age. Mean (standard error of the mean (SEM)) age of all healthy controls (39.3 years (2.7)) was not significantly different to the whole patient group ( 45.4 years (1.9)). There were no significant differences in sex.

Among the 63 patients, 20 (32\%) presented with a clinical diagnosis of fibromyalgia; 24 (38\%) presented with a clinical diagnosis of ME/CFS; and 19 (30\%) presented with dual diagnoses of fibromyalgia and ME/CFS.

On research diagnostic evaluation, 56 (89\%) met ACR diagnostic criteria for fibromyalgia, 59 (94\%) met Canadian criteria for ME/ CFS and 61 (97\%) met Fukada criteria for ME/CFS. Strikingly, $52(85 \%)$ patients met all three diagnostic criteria for fibromyalgia and ME/CFS. Seven (29\%) healthy controls had generalised joint laxity as determined by Beighton score of four or more out of nine, compared with $29(46 \%)$ in the patient group. Nine (37\%) healthy controls met Brighton criteria, compared with 51 (81\%) 
of the patient group. Two (8\%) of healthy controls met 2017 hEDS criteria, compared with 11 (18\%) of the patients. Across all participants meeting Brighton criteria, $13(22 \%)$ endorsed a hEDS diagnosis. Of those assessed as having symptomatic hypermobility only 12 (23.5\%) had received a diagnosis of hypermobility prior to evaluation in this study.

\section{Relevance of symptomatic hypermobility to diagnosis}

\section{Beighton score}

Across all participants, there were no associations between current (ie present) Beighton score and baseline pain, fatigue and interoceptive questionnaires.

Historical Beighton score (ie ever being able to perform the relevant manoeuvre) correlated (Spearman rank correlations) positively with total pain on McGill pain short form questionnaire $(r=0.25 ; n=73 ; p=0.03$ ), widespread pain index (derived from ACR diagnostic criteria; $r=0.26 ; n=86 ; p=0.01$ ), ACR symptom severity $(r=0.27 ; n=85 ; p=0.01)$, fatigue impact $(r=0.29 ; n=56 ; p=0.028)$, interoceptive sensibility $(r=0.30 ; \mathrm{n}=56 ; \mathrm{p}=0.02)$.

There was a significant interaction of being a patient with fibromyalgia and/or ME/CFS compared with being a control on the relationship between historical Beighton score and McGill pain score $(F=5.50 ; p=0.022)$; widespread pain index $(F=36.87$; $p<0.001)$; ACR symptom severity $(F=59.74 ; p \leq 0.001)$; fatigue severity scale $(F=35.29 ; p \leq 0.001)$; fatigue impact $(F=27.37$; $p \leq 0.001)$; modified fatigue impact scale $(F=37.01 ; p \leq 0.001)$; mental clutter scale $(F=15.72 ; p \leq 0.001$; univariate general linear model). This means that the relationship symptoms and being a patient and control was significantly different according to hypermobility score.

\section{Brighton criteria for joint hypermobility syndrome}

\section{Mixed fibromyalgia/ME/CFS}

Meeting Brighton criteria significantly predicted membership of the patient group (odds ratio (OR) 7.08; $\mathrm{p} \leq 0.001 ; 95 \%$ confidence interval (CI) 2.50-20.00), and meeting both major criteria for the Brighton criteria was significantly associated with being a patient (chi squared 9.26; $p=0.022$ ).

Easy bruising was significantly associated with being a patient rather than a control (chi squared 4.10; $p=0.043$ ). No minor criteria were significantly predictive of group membership. There was a significant interaction of meeting Brighton criteria on the relationship between interoceptive sensibility and group membership $(F=8.75 ; p=0.005)$.

\section{Fibromyalgia}

Endorsing both major criteria of Brighton criteria was significantly associated with an ACR fibromyalgia diagnosis (chi squared 6.53; $p=0.01$ ). ACR fibromyalgia was associated with easy bruising, (chi squared 7.58; $p=0.006$ ). No minor criteria were significantly predictive of fibromyalgia.

\section{ME/CFS}

Endorsing both major criteria of Brighton criteria was significantly associated with fulfilling Canadian criteria for ME/CFS (chi squared 7.58; $p=0.006$ ), as was endorsing Fukada criteria (chi squared $10.50, p=0.001$ ). Easy bruising (chi squared $4.22 ; p=0.04$ ) was associated with both Canadian criteria for ME/CFS and Fukada criteria (chi squared 5.71; $p=0.017$ ). No minor criteria were significantly predictive of ME/CFS.

Meeting all three diagnostic criteria (ACR, Canadian, Fukada) Meeting Brighton criteria for JHS predicted meeting all three patient diagnostic criteria ( $p<0.001$; OR 8.57; 95\% CI 3.02-24.05).

\section{hEDS 2017 diagnostic criteria}

\section{Mixed fibromyalgia and/or ME/CFS}

A diagnosis of hEDS did not predict presence of fibromyalgia and/ or ME/CFS, nor did individual elements of the diagnostic criteria.

\section{Fibromyalgia}

There were no associations between ACR definition of fibromyalgia and hEDS diagnosis or elements of the diagnostic criteria.

\section{ME/CFS}

Endorsing hEDS criterion 1 (age/sex adjusted Beighton score) significantly predicted presence of ME/CFS as defined by Canadian criteria ( $p=0.045$; OR 3.07; 95\% CI 1.01-9.28) and the Fukada criteria ( $p=0.031$; OR 3.70; $95 \%$ CI 1.12-12.18). No other elements of the hEDS diagnostic criteria were significantly predictive.

\section{Meeting all three diagnostic criteria (ACR, Canadian, Fukada)} A diagnosis of hEDS did not significantly predict meeting all three criteria. Neither did any diagnostic element.

\section{Discussion}

The strength of this research study is that it is the first clinical evaluation, to our knowledge, that incorporates and directly compares Brighton (JHS) and hEDS criteria. This study demonstrates the overlapping nature of fibromyalgia and ME/CFS within current diagnostic criteria, which remains controversial; fibromyalgia pain symptoms were recently reported in an overwhelming majority of patients with ME/CFS, the same authors also show high rates of generalized joint laxity in that population., 5,13 This shared finding is perhaps not unsurprising given the presence of pain in the Canadian criteria for ME/CFS, however, no pain level is mandated, and pain is not required if symptoms started after an infection. Nomenclature and diagnostic classification of ME/CFS are subject to ongoing debate. ${ }^{26,27}$

Importantly we show the contribution of symptomatic joint hypermobility and variants of connective tissue disorder to both conditions and to associated level of symptoms. Although we use a wide variety of patient reported outcomes, and such subjective measures can be problematic, it is important to note the significant associations between historical hypermobility and key symptoms of pain and fatigue that were not present for current hypermobility. ${ }^{28}$ In particular, ACR widespread pain index and ACR symptom severity scale allow for highly accurate identification and differentiation of fibromyalgia patients. ${ }^{29}$

Our findings of high rates of generalised joint laxity in this population are in line with other studies of ME/CFS, however, ours is the first to directly compare diagnostic assessments for hypermobility. ${ }^{13}$ Fibromyalgia and ME/CFS are both associated 
with inflammatory abnormalities and dysautonomia (eg orthostatic intolerance, postural orthostatic tachycardia syndrome and orthostatic hypotension), which is also commonly observed in symptomatic hypermobility. ${ }^{7-10}$ In fact, autonomic dysfunction is proposed to mediate disabling symptoms in fibromyalgia and has an established association with variants of connective tissue and heightened interoceptive sensibility. ${ }^{30}$ Interoceptive sensibility is the subjective measurement of the sense relating to one's own internal bodily sensations. ${ }^{24}$ While it is clear that there are associations between symptomatic hypermobility and both fibromyalgia and ME/CFS, the relationships between these conditions remain poorly understood. Ongoing research seeks to extend our observations reported here to determine how symptomatic hypermobility relates to autonomic and inflammatory induced changes in pain and fatigue in fibromyalgia and ME/CFS. Limitations of the present study include relatively small control group and difficulty in discriminating between painpredominant and fatigue-predominant disease.

This paper adds further recognition to the high rates of hypermobility in fibromyalgia and ME/CFS, which is important with growing recognition of 'post-viral' fatigue syndromes in COVID-19. However, ours is the first study to use multiple complementary assessments of joint hypermobility and variant connective tissue to explore these relationships. A rich debate has been stimulated by a recent paper reporting the prevalence of EDS and JHS. ${ }^{21}$ While other forms of EDS are likely rare, the present study suggests hEDS is common among patients with pain and fatigue. Our control group is too small to make more than anecdotal statements regarding hEDS prevalence. However, approximately one in five of those meeting Brighton criteria for joint hypermobility syndrome might endorse a diagnosis of hEDS and many patients appear to remain undiagnosed.

The classification of symptomatic joint hypermobility and variants of connective tissue is an evolving area. ${ }^{17,19,31}$ Importantly, this study reveals that distinct aspects of the diagnostic criteria for symptomatic hypermobility predict symptom severity in pain and fatigue conditions. From these data, it would appear that the Brighton criteria predict fibromyalgia and ME/CFS diagnosis and symptomatology. Interestingly, the Brighton criteria make use of the historical account of joint laxity, which was highly associated with symptom severity, compared with current joint laxity, which is not included within the hEDS 2017 criteria (criterion 1).

This relatively small study highlights the need for better recognition and accurate diagnosis in these poorly understood conditions. Fibromyalgia patients may wait, on average, almost a year after experiencing symptoms before presenting to a physician, and a diagnosis of fibromyalgia may take around 2.3 years, presenting to 3.7 different physicians, to be established. ${ }^{32}$ Once confirmed, a fibromyalgia diagnosis may have mixed benefits for patients. Similar delays to diagnosis are observed for ME/CFS. ${ }^{33}$ The impact on quality of life in fibromyalgia and ME/CFS is severe. Greater awareness among the scientific and medical community about the overlapping nature of these conditions and their system-wide symptomatology will improve the appropriate diagnosis and multi-disciplinary treatment for patients. ${ }^{34}$ Studies underway will determine the wider role of variant connective tissue (of which joint hypermobility may be but a single manifestation) and its relationship to autonomic and inflammatory induced changes in pain and fatigue in fibromyalgia and ME/CFS including interoceptive and neural mechanisms and the role of the transcriptome.

\section{Summary}

\section{What is known?}

Fibromyalgia and ME/CFS are poorly understood conditions with overlapping symptoms. Complexity may have contributed to consideration of both as functional or somatic disorders. Shared symptoms have fuelled debate as to whether they are manifestations of the same spectrum or separate entities. Both are associated with hypermobility.

\section{What is question?}

What is the contribution of variants in connective tissue (eg symptomatic joint hypermobility) to chronic pain and fatigue in fibromyalgia and ME/CFS? To what degree do these conditions overlap?

\section{What was found?}

From a research perspective, fibromyalgia and ME/CFS are almost indistinguishable. Variant connective tissue is highly prevalent and frequently overlooked in fibromyalgia and ME/CFS. Aspects of hypermobility diagnostic classification, particularly historical joint laxity, predict pain and fatigue symptomatology in these conditions.

\section{What is implication for practice now?}

All physicians will be facing the challenge of assessing possible 'post-viral fatigue' in the wake of COVID-19. Patients presenting with pain and fatigue should be assessed for hypermobility, particularly historical joint laxity in order to help inform multidisciplinary treatment targets and consideration of known comorbidities (eg autonomic dysfunction).

\section{Supplementary material}

Additional supplementary material may be found in the online version of this article at www.rcpjournals.org/clinmedicine: S1 - Diagnostic criteria to evaluate symptomatic hypermobility.

\section{Acknowledgements}

We wish to acknowledge the invaluable practical assistance of Valentina Toska and Mel Smith, the significant support of the Clinical Investigations Research Unit (Brighton and Sussex University Hospitals NHS Trust) and the editorial assistance of Robert Simonoff and AS Chamings. We wish to acknowledge the assistance of the Brighton and Sussex University NHS Hospitals Trusts Patient and Public Involvement Panel (JAFFA Panel).

\section{Funding}

This project was primarily funded by a Versus Arthritis grant (21994), with additional support provided by a studentship awarded by Action for ME and match-funded by Brighton and Sussex Medical School. The work was also supported by a charitable donation from the Fibroduck Foundation. Jessica Eccles was supported by the National Institute for Health Research (CL2015-27-002). We are indebted to the Sussex ME/CFS Society and ReMEmber for their promotion and engagement with the study.

\section{References}

1 Jones GT, Atzeni F, Beasley M et al. The prevalence of fibromyalgia in the general population: a comparison of the American 
College of Rheumatology 1990, 2010, and modified 2010 classification criteria. Arthritis Rheumatol 2015;67:568-75.

2 Katz RS, Heard AR, Mills M et al. The prevalence and clinical impact of reported cognitive difficulties (fibrofog) in patients with rheumatic disease with and without fibromyalgia. J Clin Rheumatol 2004:10:53-8.

3 Hoffman D, Dukes $E$. The health status burden of people with fibromyalgia: a review of studies that assessed health status with the SF-36 or the SF-12. Int J Clin Pract 2008;62:115-26.

4 Gerwyn M, Maes M. Mechanisms explaining muscle fatigue and muscle pain in patients with myalgic encephalomyelitis/chronic fatigue syndrome (ME/CFS): A review of recent findings. Curr Rheumatol Rep 2017;19:1.

5 Hyland ME, Bacon AM, Lanario JW et al. Symptom frequency and development of a generic functional disorder symptom scale suitable for use in studies of patients with irritable bowel syndrome, fibromyalgia syndrome or chronic fatigue syndrome. Chronic Dis Transl Med 2019;5:129-38.

6 Natelson BH. Myalgic encephalomyelitis/chronic fatigue syndrome and fibromyalgia: definitions, similarities, and differences. Clin Ther 2019;41:612-8.

7 Backryd E, Tanum L, Lind AL et al. Evidence of both systemic inflammation and neuroinflammation in fibromyalgia patients, as assessed by a multiplex protein panel applied to the cerebrospinal fluid and to plasma. J Pain Res 2017;10:515-25.

8 Missailidis D, Annesley SJ, Fisher PR. Pathological mechanisms underlying myalgic encephalomyelitis/chronic fatigue syndrome. Diagnostics (Basel) 2019;9:80

9 Newton JL, Okonkwo O, Sutcliffe K et al. Symptoms of autonomic dysfunction in chronic fatigue syndrome. QJM 2007;100:519-26.

10 De Wandele I, Rombaut L, Leybaert L et al. Dysautonomia and its underlying mechanisms in the hypermobility type of Ehlers-Danlos syndrome. Semin Arthritis Rheum 2014;44:93-100.

11 Castori M, Celletti C, Camerota F et al. Chronic fatigue syndrome is commonly diagnosed in patients with Ehlers-Danlos syndrome hypermobility type/joint hypermobility syndrome. Clin Exp Rheumatol 2011:29:597-8.

12 Rowe PC, Barron DF, Calkins $\mathrm{H}$ et al. Orthostatic intolerance and chronic fatigue syndrome associated with Ehlers-Danlos syndrome. J Pediatr 1999:135:494-9.

13 Bragée B, Michos A, Fahlgren M, Szulkin R, Bertilson BC. Signs of intracranial hypertension, hypermobility, and craniocervical obstructions in patients with myalgic encephalomyelitis/chronic fatigue syndrome. Front Neurol 2020;11:828.

14 Grahame R. Hypermobility: an important but often neglected area within rheumatology. Nat Clin Pract Rheumatol 2008;4:522-4.

15 Mulvey MR, Macfarlane G], Beasley M et al. Modest association of joint hypermobility with disabling and limiting musculoskeletal pain: results from a large-scale general population-based survey. Arthritis Care Res (Hoboken) 2013;65:1325-33.

16 Castori M. Ehlers-Danlos syndrome, hypermobility type: an underdiagnosed hereditary connective tissue disorder with mucocutaneous, articular, and systemic manifestations. ISRN Dermatol 2012;2012:751768.

17 Castori M, Tinkle B, Levy $\mathrm{H}$ et al. A framework for the classification of joint hypermobility and related conditions. Am J Med Genet $C$ Semin Med Genet 2017;175:148-57.

18 Grahame R, Bird HA, Child A. The revised (Brighton 1998) criteria for the diagnosis of benign joint hypermobility syndrome (BJHS). J Rheumatol 2000:27:1777-9.
19 Tinkle BT, Bird HA, Grahame R et al. The lack of clinical distinction between the hypermobility type of Ehlers-Danlos syndrome and the joint hypermobility syndrome (a.k.a. hypermobility syndrome). Am J Med Genet A 2009;149A:2368-70.

20 Copetti M, Morlino S, Colombi M et al. Severity classes in adults with hypermobile Ehlers-Danlos syndrome/hypermobility spectrum disorders: a pilot study of 105 Italian patients. Rheumatology (Oxford) 2019;58:1722-30.

21 Demmler JC, Atkinson MD, Reinhold EJ et al. Diagnosed prevalence of Ehlers-Danlos syndrome and hypermobility spectrum disorder in Wales, UK: a national electronic cohort study and case-control comparison. BMJ Open 2019;9:e031365.

22 Junge T, Henriksen P, Hansen S et al. Generalised joint hypermobility and knee joint hypermobility: prevalence, knee joint symptoms and health-related quality of life in a Danish adult population. Int J Rheum Dis 2019;22:288-96.

23 Ofluoglu D, Gunduz OH, Kul-Panza E et al. Hypermobility in women with fibromyalgia syndrome. Clin Rheumatol 2006;25:291-3.

24 Garfinkel SN, Seth AK, Barrett AB et al. Knowing your own heart: distinguishing interoceptive accuracy from interoceptive awareness. Biological psychology 2015;104:65-74.

25 Paepe AD, Malfait F. Bleeding and bruising in patients with Ehlers-Danlos syndrome and other collagen vascular disorders. $\mathrm{Br}]$ Haematol 2004:127:491-500.

26 Jason LA, Sunnquist M, Brown A et al. Chronic fatigue syndrome versus systemic exertion intolerance disease. Fatigue 2015;3:127-41.

27 Asprusten TT, Sulheim D, Fagermoen E et al. Systemic exertion intolerance disease diagnostic criteria applied on an adolescent chronic fatigue syndrome cohort: evaluation of subgroup differences and prognostic utility. BMJ Paediatr Open 2018;2:e000233.

28 Haywood KL, Staniszewska S, Chapman S. Quality and acceptability of patient-reported outcome measures used in chronic fatigue syndrome/myalgic encephalomyelitis (CFS/ME): a systematic review. Qual Life Res 2012;21:35-52.

29 Galvez-Sánchez CM, de la Coba P, Duschek S et al. Reliability, factor structure and predictive validity of the widespread pain index and symptom severity scales of the 2010 American College of Rheumatology criteria of fibromyalgia. J Clin Med 2020;9:2460.

30 Aslanyan D, Iodice V, Davies KA et al. Dissociative experiences in fibromyalgia are mediated by symptoms of autonomic dysfunction. medRxiv 2019:19006320.

31 Castori M, Hakim A. Contemporary approach to joint hypermobility and related disorders. Curr Opin Pediatr 2017;29:640-49.

32 Choy E, Perrot S, Leon T et al. A patient survey of the impact of fibromyalgia and the journey to diagnosis. BMC Health Serv Res 2010;10:102.

33 Comiskey C, Larkan F. A national cross-sectional survey of diagnosed sufferers of myalgic encephalomyelitis/chronic fatigue syndrome: pathways to diagnosis, changes in quality of life and service priorities. Ir J Med Sci 2010;179:501-5.

34 Royal College of General Practitioners. The Ehlers-Danlos syndromes toolkit. RCoG. www.rcgp.org.uk/eds

Address for correspondence: Dr Jessica A Eccles, Trafford Centre for Medical Research, University of Sussex, Falmer, Brighton BN1 9RY, UK.

Email: j.eccles@bsms.ac.uk

Twitter: @BendyBrain 